gr-qc/9803035

\title{
Wormhole effective interactions in anti-de Sitter spacetime
}

\author{
Carlos Barceló ${ }^{1,2}$ and Luis J. Garay ${ }^{2}$ \\ ${ }^{1}$ Instituto de Astrofísica de Andalucía, CSIC \\ Camino Bajo de Huétor, 18080 Granada, Spain \\ 2 Instituto de Matemáticas y Física Fundamental, CSIC \\ C/ Serrano 121, 28006 Madrid, Spain
}

10 March 1998

\begin{abstract}
The effects of asymptotically anti-de Sitter wormholes in low-energy field theory are calculated in full detail for three different matter contents: a conformal scalar field, an electromagnetic field and gravitons. There exists a close relation between the choice of vacuum for the matter fields and the selection of a basis of the Hilbert space of anti-de Sitter wormholes. In the presence of conformal matter (i.e., conformal scalar or electromagnetic fields), this relation allows us to interpret the elements of these bases as wormhole states containing a given number of particles. This interpretation is subject to the same kind of ambiguity in the definition of particle as that arising from quantum field theory in curved spacetime. In the case of gravitons, owing to the non-conformal coupling, it is not possible to describe wormhole states in terms of their particle content.
\end{abstract}

PACS: 04.60.Ds, 04.62.+v, 98.80 Hw

Keywords: anti-de Sitter wormholes, effective interactions, harmonic expansion

\section{Introduction}

Wormholes describe processes that involve baby universes branching off and joining onto asymptotically large regions of spacetime and have been extensively studied in the literature [1]. Their effects in low-energy fields can be taken into account, in the dilute wormhole approximation, by introducing local effective interactions in a fixed background [2, 3]. As a consequence, wormholes would modify the coupling constants of any low-energy effective physical theory. These effective interactions produced by wormholes have been calculated for a variety of matter fields coupled to gravity [4, 5, 6, 0, 8, 9]. In all these calculations, the asymptotically large region of the wormhole has been assumed to be flat. Therefore, the effective interactions produced by such wormholes have been modelled by interacting terms added to a free quantum field theory in flat spacetime.

In Refs. [10, 11], we dealt with wormholes with other types of asymptotic behaviour; in particular, we studied the case of wormholes in asymptotically anti-de Sitter (adS) spacetimes. This exhausts all possible maximally symmetric asymptotic behaviours. Also, adS wormholes could be regarded as excited states in the sense that the cosmological constant $\Lambda$ could be interpreted as a non-vanishing asymptotic energy of the matter field.

Wormholes in flat spacetime do not contribute to the cosmological constant nor to the gravitational coupling constant $G$ directly [3, 7]. In Ref. [11], we showed that this is 
also true in adS spacetime. Furthermore, the interaction Lagrangians for the lower inhomogeneous modes in adS spacetime do not have any contribution from the cosmological constant and therefore have the same form as in the flat case. However, the interaction Lagrangians for the higher inhomogeneous modes in adS spacetime and those in the flat case differ in terms that are proportional to powers of the cosmological constant. In the case of gravitons, the non-conformal nature of their coupling, although present, is hidden in the flat case but explicit in adS spacetimes. This introduces new elements in the analysis of the latter case, such as the non-separability between the homogeneous and inhomogeneous modes. Another interesting characteristic of this model is that there naturally exist different possible vacuum choices for the matter fields in the asymptotic regions, as happens in quantum field theory in curved spacetime. We saw in Ref. [11], and will be further discussed here, that there exists a relation between the definition of this vacuum and the choice of basis in the Hilbert space of quantum wormholes, which allows us, for conformally invariant matter, to interpret the basis elements as states of wormholes containing certain number of particles.

In this paper, we will consider three separate kinds of matter fields coupled to gravity in the presence of a negative cosmological constant, namely, a conformal scalar field, an electromagnetic field and gravitons. We will model the effects of closed universes that branch off or join onto an asymptotically adS spacetime in the dilute wormhole approximation by means of an effective quantum field theory in an adS background [2, 12. In order to find these effects on the matter fields, and following previous analyses [4, 5, 6, 7, 8, 9, 11], we will explicitly calculate matrix elements $\left\langle 0\left|\Phi\left(x_{1}\right) \cdots \Phi\left(x_{r}\right)\right| \Psi_{\alpha}\right\rangle$ of products of operators $\Phi(x)$, each representing a generic matter field at a different point, between an specific vacuum $|0\rangle$ and the elements $\left\{\Psi_{\alpha}\right\}$ of a basis of the Hilbert space of adS wormholes [4]. In flat spacetime, the state that is void of particles for inertial observers defines a preferred vacuum for which its associated propagator is asymptotically damped. In adS spacetime, we can define a one-parameter family of maximally symmetric vacua by analogy with the de-Sitter case 114, 15. The propagator associated with each vacuum of this family is damped when the geodesic distance between the two points becomes large, but with a different fall-off for each vacuum choice [16]. This family of vacua is the analogue in adS spacetime of the vacua defined by uniformly accelerated reference frames in the flat case. The state $|0\rangle$ is any fixed vacuum of this family.

In order to calculate the local effective interaction terms produced by adS wormholes, we need to obtain the quantum wormhole wave functions for these systems. This will be done in Sec. 2. Section 3 is devoted to calculate semiclassically the two-point functions for each matter content in the presence of a wormhole. Processes involving more complicated matrix elements could be treated in a similar way. We will find the effective interaction terms that have to be added to the bare action in order to reproduce these Green functions in Sec. 4. Finally, we will summarise the results of this work and conclude in Sec. 5 .

\footnotetext{
1 There are some subtleties in the definition of states in an adS Lorentzian background because this spacetime does not posses a well-posed Cauchy problem. However, we will work in the Euclidean sector where this issue does not arise 13].
} 


\section{Wormhole wave functions}

In order to find the wormhole wave functions, we have to solve the WDW equation $\mathcal{H} \Psi_{\alpha}=0$ and the momenta constraint equations $\mathcal{H}_{i} \Psi_{\alpha}=0$ with appropriate boundary conditions. These quantum constraints are associated with the invariance of general relativity under time reparametrisations (modulo spatial diffeomorphisms) and spatial diffeomorphisms, respectively. They are difficult to solve exactly and, therefore, we will perform a perturbative treatment. We will take a homogeneous and isotropic spacetime, characterised by its scale factor, which will be restricted to be positive in order to avoid repetition of geometries, and the homogeneous mode of the matter field as the configurations variables that will be treated exactly. Then, we will decompose the perturbation around the homogeneous configuration in hypersherical harmonics on the three-sphere $S^{3}$. The coefficients of this expansion will represent the rest of the degrees of freedom.

\subsection{Conformal scalar field}

Let us now consider a scalar field conformally coupled to gravity. Perturbatively, we can express the scalar field $\phi$ and the spatial part of the metric $g_{i j}$ as?

$$
\begin{gathered}
\phi=\sqrt{\frac{1}{2 \pi^{2}}} a^{-1} \sum_{n, \sigma_{n}} \chi_{n \sigma_{n}} Q^{n \sigma_{n}}, \\
g_{i j}=a^{2} \Omega_{i j}+h_{i j}, \\
h_{i j}=\sum_{n, \sigma_{n}}\left(\sqrt{6} a_{n \sigma_{n}} Q_{i j}^{n \sigma_{n}}+\sqrt{6} b_{n \sigma_{n}} P_{i j}^{n \sigma_{n}}+\sqrt{2} c_{n \sigma_{n}} S_{i j}^{n \sigma_{n}}+2 d_{n \sigma_{n}} G_{i j}^{n \sigma_{n}}\right),
\end{gathered}
$$

$\Omega_{i j}$ being the metric in the unit three-sphere. The hyperspherical harmonics are defined as follows [17]. $Q^{n \sigma_{n}}, S_{i}^{n \sigma_{n}}$ and $G_{i j}^{n \sigma_{n}}$ are the scalar, transverse vector $\left(S_{i}^{n \sigma_{n} \mid i}=0\right)$ and transverse traceless tensor $\left(G_{i j}^{n \sigma_{n} \mid j}=0, G_{i}^{n \sigma_{n} i}=0\right)$ harmonics. They are eigenfunctions of the Laplace-Beltrami operator, $\Omega^{i j} \nabla_{i} \nabla_{j}$, in $S^{3}$ with eigenvalues $-\left(n^{2}-1\right),-\left(n^{2}-2\right)$ and $-\left(n^{2}-3\right)$, respectively. The index $\sigma_{n}$ runs over a basis of the corresponding degenerate eigenspace. The remaining tensor harmonics are defined as:

$$
Q_{i j}^{n \sigma_{n}}=\Omega_{i j} \frac{1}{3} Q^{n \sigma_{n}}, \quad P_{i j}^{n \sigma_{n}}=\frac{1}{\left(n^{2}-1\right)} Q_{\mid i j}^{n \sigma_{n}}+Q_{i j}^{n \sigma_{n}}, \quad S_{i j}^{n \sigma_{n}}=S_{(i \mid j)}^{n \sigma_{n}} .
$$

Among the degrees of freedom that we are considering, the coefficients $a_{n \sigma_{n}}, b_{n \sigma_{n}}$ and $c_{n \sigma_{n}}$ are gauge; we can set them to zero by means of an appropriate diffeomorphism in $S^{3}$ and introducing suitable lapse and shift functions. The variables $d_{n \sigma_{n}}$, however, are true degrees of freedom associated with gravitational waves. For the time being, we set them to zero, $d_{n \sigma_{n}}=0$.

Then, the WDW equation becomes

$$
\left[-\frac{\partial^{2}}{\partial a^{2}}+a^{2}+\lambda a^{4}-\sum_{n, \sigma_{n}}\left(-\frac{\partial^{2}}{\partial \chi_{n \sigma_{n}}^{2}}+n^{2} \chi_{n \sigma_{n}}^{2}-\frac{1}{2}\right)\right] \Psi\left(a, \chi_{n \sigma_{n}}\right)=0 .
$$

\footnotetext{
${ }^{2}$ Throughout this paper, we will use a rescaled cosmological constant $\lambda=-\frac{1}{3} \Lambda$ and set $\frac{3}{2} \pi m_{p}^{2}=1$.
} 
In the process of arriving at the WDW equation, we have chosen an operator ordering that removes the ground-state energy of each oscillator. Its solutions can be obtained by separation of variables:

$$
\begin{gathered}
{\left[\sum_{n, \sigma_{n}}\left(-\frac{\partial^{2}}{\partial \chi_{n \sigma_{n}}^{2}}+n^{2} \chi_{n \sigma_{n}}^{2}-\frac{1}{2}\right)\right] \varphi_{E}\left(\chi_{n \sigma_{n}}\right)=E \varphi_{E}\left(\chi_{n \sigma_{n}}\right),} \\
{\left[-\frac{\partial^{2}}{\partial a^{2}}+a^{2}+\lambda a^{4}\right] \psi_{E}(a)=E \psi_{E}(a) .}
\end{gathered}
$$

We impose the standard boundary conditions for quantum harmonic oscillators to the matter field part $\varphi_{E}$, so that $E$ will be a sum of harmonic oscillator energies. As for the gravitational sector, we will only demand that $\psi_{E}$ is exponentially damped when $a \rightarrow+\infty$. These boundary conditions and their connection with the path integral formalism were studied in detail in Ref. [10]. The wave functions must be further restricted, owing to the linearisation instability of the perturbations to the rotationally invariant homogeneous configuration $\left(a, \chi_{1}\right)[7,[18]$. This restriction should make the wave functions depend on the inhomogeneous $n>1$ configuration variables only through the rotationally invariant combination $\chi_{n}^{2}=\sum_{\sigma_{n}} \chi_{n \sigma_{n}}^{2}$.

We finally obtain wave functions of the form

$$
\Psi_{N_{1}, \cdots, N_{n}, \cdots}\left(a, \chi_{n}\right)=\psi_{E}(a) \mathcal{H}_{N_{1}}\left(\chi_{1}\right) e^{-\frac{1}{2} \chi_{1}^{2}} \prod_{n>1} \mathcal{L}_{N_{n}}^{\left(n^{2}-3\right) / 2}\left(n \chi_{n}^{2}\right) e^{-\frac{1}{2} n \chi_{n}^{2}},
$$

where $E=N_{1}+\sum_{n>1} 2 n N_{n}, \mathcal{H}_{N_{1}}$ is the Hermite polynomial of degree $N_{1}$ and $\mathcal{L}_{N_{n}}^{\left(n^{2}-3\right) / 2}$ is the generalised Laguerre polynomial of degree $N_{n}$ in its arguments [19]. These wave functions form an orthonormal basis of the Hilbert space of wormhole quantum states with the measure $\int d a \prod_{n, \sigma_{n}} d \chi_{n \sigma_{n}}$, which is proportional to

$$
\int_{0}^{+\infty} d a \int_{-\infty}^{+\infty} d \chi_{1} \prod_{n>1} \int_{0}^{+\infty} d \chi_{n}\left(\chi_{n}\right)^{n^{2}-1}
$$

owing to the rotational invariance. The choice of this measure can be justified in the context of the algebraic quantization program (see Ref. [10]). Indeed, it is gauge dependent, but this spurious dependence can be absorbed in the normalization constants and therefore does not affect the results. Note that, with this choice, the orthogonality relations are dictated by the matter field part of the measure.

We can interpret $N_{1}$ as the number of homogeneous scalar particles inside the wormhole. A wave function with vanishig $N_{n}$ 's except for a single mode $n_{0}>1$ such that $N_{n_{0}}=1$, can be interpreted as a rotationally invariant superposition of two-particle states in the mode $n_{0}$. Then, $N_{n}$, with $n>1$, will be the number of this kind of two-particle states inside the wormhole. As we will see in Sec. 3, these particles must be associated with a specific vacuum, which will be the one conformally related to the natural vacuum for flat spacetimes.

\subsection{Electromagnetic field}

We can decompose the electromagnetic field $A_{\mu}$ in harmonics in the three-sphere:

$$
A_{0}=\sqrt{\frac{1}{2 \pi^{2}}} \sum_{n, \sigma_{n}} \alpha_{n \sigma_{n}} Q^{n \sigma_{n}}, \quad A_{i}=\sqrt{\frac{1}{2 \pi^{2}}} \sum_{n, \sigma_{n}}\left(\beta_{n \sigma_{n}} S_{i}^{n \sigma_{n}}+\gamma_{n \sigma_{n}} P_{i}^{n \sigma_{n}}\right),
$$


where the harmonics $Q^{n \sigma_{n}}, S_{i}^{n \sigma_{n}}$ were introduced in the previous subsection and the longitudinal vector harmonics $P_{i}^{n \sigma_{n}}$ are defined through their relation with the scalar ones $P_{i}^{n \sigma_{n}}=\frac{1}{n^{2}-1} Q_{\mid i}^{n \sigma_{n}}$.

The wave functions do not depend on $\alpha_{n \sigma_{n}}$ and $\gamma_{n \sigma_{n}}$ because these are Lagrange multipliers [6]. They only depend on the transverse degrees of freedom $\beta_{n \sigma_{n}}$. Therefore, the WDW equation for electromagnetic wormholes in adS spacetime can be written as

$$
\left[-\frac{\partial^{2}}{\partial a^{2}}+a^{2}+\lambda a^{4}-\sum_{n, \sigma_{n}}\left(-\frac{\partial^{2}}{\partial \beta_{n \sigma_{n}}^{2}}+n^{2} \beta_{n \sigma_{n}}^{2}-\frac{1}{2}\right)\right] \Psi\left(a, \beta_{n \sigma_{n}}\right)=0 .
$$

The transverse degrees of freedom can be separated into positive and negative helicities because the helicity operator commutes with rotations. Their respective coefficients in the harmonic expansion will be called $\beta_{n \sigma_{n}}^{ \pm}$. The requirement that the wave functions must be invariant under $S O(4)$ implies that they only depend on the invariant quantities $\left(\beta_{n}^{ \pm}\right)^{2}=\sum_{\sigma_{n}}\left(\beta_{n \sigma_{n}}^{ \pm}\right)^{2}$, where $\sigma_{n}$ runs over the corresponding positive and negative helicity degenerate eigenspaces. As in the scalar field case, an orthonormal basis of the Hilbert space of rotationally invariant wave functions for this system is given by

$$
\Psi_{N_{2}^{ \pm}, \cdots, N_{n}^{ \pm}, \ldots}\left(a, \beta_{n}^{ \pm}\right)=\psi_{E}(a) \prod_{n, p= \pm} \mathcal{L}_{N_{n}^{p}}^{\left(n^{2}-4\right) / 2}\left(n\left(\beta_{n}^{p}\right)^{2}\right) e^{-\frac{1}{2} n\left(\beta_{n}^{p}\right)^{2}}
$$

where $E=\sum_{n=2} 2 n\left(N_{n}^{+}+N_{n}^{-}\right)$and $\mathcal{L}_{N_{n}^{p}}^{\left(n^{2}-4\right) / 2}$ are the Laguerre polynomials. $N_{n}^{ \pm}$can be interpreted as the number of rotationally invariant two-particle states of positive and negative helicity in the mode $n$ inside the wormhole, which are associated with the conformal vacuum as we will see in Sec 3. A wormhole cannot contain a single-particle state because of the $S O(4)$ rotational invariance. This symmetry forbids the existence of wormholes with non-vanishing global spin.

\subsection{Gravitational perturbations}

The last case we are going to consider is that of gravitons, represented by the coefficients $d_{n \sigma_{n}}$ of the transverse traceless tensor harmonics $G_{i j}^{n \sigma_{n}}$ in the expansion of the metric (2.3). We expand the constraints up to second order in perturbation theory and, thus, the dynamical equations for $d_{n \sigma_{n}}$, obtained by varying these constraints, will be first order in $d_{n \sigma_{n}}$. It has been shown [20] that, when the Einstein tensor of the background spacetime is traceless, the behaviour of gravitational waves in that background is similar to that of a conformal scalar field. For this reason, the WDW equation in the flat case splits into two separate parts [7]. However, if the cosmological constant does not vanish, the trace of the Einstein tensor is $-6 \lambda$ and, therefore, the above argument does not apply. In fact, we can write the WDW equation for a non-vanishing cosmological constant as

$$
\left[-\frac{\partial^{2}}{\partial \tilde{a}^{2}}+\tilde{a}^{2}+\lambda \tilde{a}^{4}-\sum_{n, \sigma_{n}}\left(-\frac{\partial^{2}}{\partial \tilde{d}_{n \sigma_{n}}^{2}}+n^{2} \tilde{d}_{n \sigma_{n}}^{2}+2 \lambda \tilde{a}^{2} \tilde{d}_{n \sigma_{n}}^{2}\right)\right] \Psi\left(\tilde{a}, \tilde{d}_{n \sigma_{n}}\right)=0
$$

where $\tilde{a}=a\left(1-3 / 2 \sum d_{n \sigma_{n}}^{2}\right), \tilde{d}_{n \sigma_{n}}=\tilde{a} d_{n \sigma_{n}}$ and we have redefined the lapse function as $\tilde{N}=N\left(1+2 \sum d_{n \sigma_{n}}^{2}\right)$. In this expression, it is understood that the vacuum energies have to be removed. 
The WDW equation is quite involved and we have not succeeded in finding explicit solutions. However, when the scale factor becomes large, the wave function will be picked around an asymptotically adS spacetime and we will then recover a quantum field theory of gravitons on the adS background (in this approximation, the back-reaction can be neglected) [21, 22]. Then, the semiclassical wave functions for the graviton part will be harmonic oscillator eigenfunctions with a frequency that depends on the scale factor [22]. These considerations allow us to construct a formal basis of the Hilbert space of this system, denoted by $\left\{\Psi_{\alpha}\left(a, d_{n}^{ \pm}\right)\right\}$, in which the requirement of rotational invariance has already been imposed.

\section{Two-point functions}

We are interested in processes in which there is creation or annihilation of particles associated with a particular vacuum in two different points of a spacetime region where a wormhole end is inserted. Following Hawking [4], we first have to calculate a path integral over geometries and matter fields that induce a metric $g_{i j}^{0}$ and a value $\Phi^{0}$ for the matter field in a cross section of the wormhole. Also, they must satisfy some asymptotic requirements that guarantee the asymptotically adS behaviour and that select a vacuum for the matter field in the semiclassical approximation. After that, we will integrate over all possible configurations $\left(g_{i j}^{0}, \Phi^{0}\right)$ weighted with the wormhole wave function, i.e.

$$
\left\langle 0\left|\Phi\left(x_{1}\right) \Phi\left(x_{2}\right)\right| \Psi_{\alpha}\right\rangle=\int \mathcal{D} \Phi^{0} \mathcal{D} g_{i j}^{0} \Psi_{\alpha}\left[g_{i j}^{0}, \Phi^{0}\right] \int \mathcal{D} \Phi \mathcal{D} g_{\mu \nu} \Phi\left(x_{1}\right) \Phi\left(x_{2}\right) e^{-I\left[g_{\mu \nu}, \Phi\right]}
$$

We will evaluate this path integral semiclassically. The action $I\left[g_{\mu \nu}, \Phi\right]$ contains a surface term that renders it finite for classical solutions [10], a necessary requirement for the semiclassical approximation to be meaningful. The saddle point solution for the gravitational part must be an asymptotically adS spacetime. As far as the low-energy regime is concerned, it can be taken to be pure adS spacetime outside a three-sphere in which the wave function takes its arguments.

The position of the spacetime points $x_{1}, x_{2}$ can only be specified modulo the isometries of Euclidean adS spacetime, $S O(4,1)$. If $\Phi(x)$ is a saddle point solution for the matter field, then, $\Phi_{g}(x)$, the transform of $\Phi$ under an element $g^{-1}$ of the group of isometries $S O(4,1)$, is also a solution. It has the form

$$
\Phi_{g}(x)=T\left(x, x_{g}\right) \Phi\left(x_{g}\right),
$$

where $x_{g}$ stands for the transform of $x$ under $g$ and $T\left(x, x_{g}\right)$ is the usual tensor transfor-

mation given by a product of factors $\frac{\partial x_{g}}{\partial x}$, one for each spacetime index of the matter field $\Phi$. As a consequence, one has to average over this group. AdS spacetime is isomorphic to the coset space $S O(4,1) / S O(4)$. A property of unimodular groups [23 tells us that integrating a function $F$ in the group $S O(4,1)$, using the invariant Haar measure in that group, is equivalent to integrating first over the group $S O(4)$ and, then, over the coset space, i.e. over adS spacetime

$$
\int_{S O(4,1)} d g F(g)=\int_{\mathrm{adS}} d^{4} x_{0} \sqrt{g\left(x_{0}\right)} \int_{S O(4)} d h F\left(x_{0} h\right)
$$


$h$ being a generic element of the isotropy group $S O(4)$. We can interpret this integral as an average over the orientations of the wormhole and the positions in which it can be inserted. If we expand the field in hyperspherical harmonics, the average over orientations must be performed independently for each mode. In flat spacetime, we obtain similar results if we use its own group of isometries, the Euclidean group in four dimensions $E_{4}$ [4].

Throughout this paper, we shall describe adS spacetime with two different sets of coordinates: polar coordinates, $\left\{\mu, \theta_{i}\right\}$, with a line element

$$
d s^{2}=d \mu^{2}+C(\mu)^{-2} d \Omega_{3}^{2}\left(\theta_{i}\right),
$$

$d \Omega_{3}^{2}\left(\theta_{i}\right)$ being the metric on the unit three-sphere and $C(\mu)=\frac{\sqrt{\lambda}}{\sinh (\sqrt{\lambda} \mu)}$, and quasiEuclidean coordinates, $\vec{x}=\left\{x^{1}, x^{2}, x^{3}, x^{4}\right\}$, for which

$$
d s^{2}=d \vec{x}^{2}-\frac{\lambda(\vec{x} \cdot d \vec{x})^{2}}{\left(1+\lambda \vec{x}^{2}\right)}
$$

where the dot represents Euclidean contraction of indices. The change of coordinates that relates these two sets is of the form $x^{a}=C(\mu)^{-1} \widehat{x}^{a}\left(\theta_{i}\right), \widehat{x}^{a}\left(\theta_{i}\right)$ being the unit vector in the direction of $x^{a}$ and thus $|\vec{x}|=C(\mu)^{-1}$.

The adS isometries can be divided into two classes: quasi-translations and rotations [24]. We will denote by $\vec{x}_{x_{0}}$ the transform of $\vec{x}$ under a quasi-translation with parameter $-\vec{x}_{0}$ [24]:

$$
\vec{x}_{x_{0}}=\vec{x}-\vec{x}_{0}\left[\left(1+\lambda \vec{x}^{2}\right)^{1 / 2}-\frac{\left[\left(1+\lambda \vec{x}_{0}^{2}\right)^{1 / 2}-1\right]}{\vec{x}_{0}^{2}}\left(\vec{x} \cdot \vec{x}_{0}\right)\right] .
$$

Note that, when $\lambda=0$, we get $\vec{x}_{x_{0}}=\vec{x}-\vec{x}_{0}$, i.e. a translation in flat space. The image of $\vec{x}$ under an $S O(4)$ rotation $h$ will be called

$$
\vec{x}_{h} \equiv R(h) \vec{x}
$$

$R(h)$ being a rotation matrix.

\subsection{Conformal scalar field}

In this subsection, we will calculate the two-point matrix element for a conformal scalar field,

$$
\left\langle 0\left|\phi\left(x_{1}\right) \phi\left(x_{2}\right)\right| \Psi_{\alpha}\right\rangle,
$$

in the semiclassical approximation. Therefore, we need to find all the saddle points for the scalar field, $\phi_{x_{0} h}$, which are characterised by their position and orientation with respect to an arbitrary reference. As remarked above, pure adS spacetime outside a three-sphere can be regarded as the gravitational saddle point. Then, the conformal scalar field solutions must satisfy the equation

$$
(\square+2 \lambda) \phi=0,
$$

where $\square$ is the Laplacian for the adS metric given by Eq. (3.4) or (3.5). In order to solve this equation, we decompose again the scalar field in hyperspherical harmonics in the three-sphere using polar coordinates,

$$
\phi(x)=a^{-1}(\mu) \sum_{n, \sigma_{n}} \chi_{n \sigma_{n}}(\mu) Q^{n \sigma_{n}}\left(\theta_{i}\right)
$$


where $a(\mu)=C(\mu)^{-1}$ is the adS scale factor. In this expression, we have dropped an

irrelevant constant factor $\sqrt{\frac{1}{2 \pi^{2}}}$. For each mode $n$, we choose a cartesian basis in the corresponding degenerate space [17], so that

$$
Q^{n \sigma_{n}}\left(\theta_{i}\right)=\mathcal{A}_{a_{1} \cdots a_{n-1}}^{n \sigma_{n}} \widehat{x}^{a_{1}} \cdots \widehat{x}^{a_{n-1}},
$$

where $\mathcal{A}^{n \sigma_{n}}$ are the elements of an orthonormal basis in the linear space of the symmetric tensors of rank $n-1$, traceless in every pair of indices. Then, Eq. (3.9) becomes a set of decoupled ordinary differential equations, one for each coefficient $\chi_{n \sigma_{n}}$,

$$
\ddot{\chi}_{n \sigma_{n}}+A(\mu) \dot{\chi}_{n \sigma_{n}}-n^{2} C^{2}(\mu) \chi_{n \sigma_{n}}=0 .
$$

Here, the overdot means derivative with respect to $\mu$ and

$$
A(\mu)=-\dot{C}(\mu) / C(\mu) \text {. }
$$

The solutions of these equations must have the same fall-off at infinity for all $\sigma_{n}$. This asymptotic behaviour depends on the choice of vacuum. As second order linear differential equations, this requirement determines one of the two arbitrary constants for each $\sigma_{n}$. The remaining constants are determined by the value of the radius of the three-sphere that is going to be identify with a section $\Sigma$ of the wormhole, $a^{0}$, and the values of the field, $\chi_{n \sigma_{n}}^{0}$, in this three-sphere. We can write the solutions as

$$
\chi_{n \sigma_{n}}(\mu)=\frac{\chi_{n \sigma_{n}}^{0}}{f_{n}\left(\mu^{0}\right)} f_{n}(\mu), \quad \mu^{0} \equiv \frac{1}{\sqrt{\lambda}} \sinh ^{-1}\left(\sqrt{\lambda} a^{0}\right) .
$$

The saddle point can then be re-expressed as

$$
\phi(x)=C(\mu) \sum_{n} f_{n}(\mu) \mathcal{A}_{a_{1} \cdots a_{n-1}}^{n} \widehat{x}^{a_{1}} \cdots \widehat{x}^{a_{n-1}},
$$

with

$$
\mathcal{A}_{a_{1} \cdots a_{n-1}}^{n}=\sum_{\sigma_{n}} \mathcal{A}_{a_{1} \cdots a_{n-1}}^{n \sigma_{n}} \frac{\chi_{n \sigma_{n}}^{0}}{f_{n}\left(\mu^{0}\right)} .
$$

Note that the tensor $\mathcal{A}^{n}$ has the same symmetries as the elements of the cartesian basis $\mathcal{A}^{n \sigma_{n}}$. Equation (3.15) is already written in quasi-Euclidean coordinates. We can obtain all the saddle points for the scalar field by translating and rotating this particular one:

$$
\phi_{x_{0} \bar{h}}(x)=C(\mu) \sum_{n} f_{n}(\mu) \mathcal{A}_{a_{1} \cdots a_{n-1}}^{n} \widehat{x}_{x_{0} h_{n}}^{a_{1}} \cdots \widehat{x}_{x_{0} h_{n}}^{a_{n-1}},
$$

where $\bar{h}=\left\{h_{1}, \cdots, h_{n}, \cdots\right\}$ is the set of independent rotations for each mode and $\mu=$ $\mu\left(x, x_{0}\right)$ is the geodesic distance between the points $x$ and $x_{0}$, defined through the relation

$$
\mu\left(x, x_{0}\right)=\frac{1}{\sqrt{\lambda}} \sinh ^{-1}\left(\sqrt{\lambda}\left|\vec{x}_{x_{0}}\right|\right) .
$$

We are now ready to approximate the matrix element (3.8) semiclassically:

$$
\begin{aligned}
& \left\langle 0\left|\phi\left(x_{1}\right) \phi\left(x_{2}\right)\right| \Psi_{N_{1}, \cdots, N_{n}, \cdots}\right\rangle=\int_{0}^{+\infty} d a \int_{-\infty}^{+\infty} d \chi_{1} \prod_{m>1} \int_{0}^{+\infty} d \chi_{m}\left(\chi_{m}\right)^{m^{2}-1} \times \\
& \int d^{4} x_{0} \sqrt{g\left(x_{0}\right)} \int_{S O(4)} d \bar{h} \phi_{x_{0} \bar{h}}\left(x_{1}\right) \phi_{x_{0} \bar{h}}\left(x_{2}\right) \Delta\left(a^{0}, \chi_{n}^{0}\right) e^{-I^{s p}\left(a^{0}, \chi_{1}^{0}\right)} \Psi_{N_{1}, \cdots, N_{n}, \cdots}\left(a, \chi_{n}^{0}\right),
\end{aligned}
$$


where $\Delta$ is the sum of the quadratic fluctuations around the classical solution and the integral over $\bar{h}$ represents and integral over each $h_{n}$ independently. Note that the wave function $\Psi$, the action $I^{s p}$ and the determinant $\Delta$ are rotationally invariant. Thus, the integrand depends on the group $S O(4)$ only through the saddle point solutions $\phi_{x_{0}} \bar{h}$.

Let us analyse each mode independently. For the lowest mode $n=1$, the saddle points depend only on $\mu$; therefore, the average over orientations has no effect and Eq. (3.19) reduces to

$$
\begin{aligned}
\left\langle 0\left|\phi\left(x_{1}\right) \phi\left(x_{2}\right)\right| \Psi_{N_{1}}\right\rangle= & \int_{0}^{+\infty} d a^{0} \int_{-\infty}^{+\infty} d \chi_{1}^{0}\left(\frac{\chi_{1}^{0}}{f_{1}\left(\mu^{0}\right)}\right)^{2} \Delta\left(a^{0}, \chi_{1}^{0}\right) e^{-I^{s p}\left(a^{0}, \chi_{1}^{0}\right)} \Psi_{N_{1}}\left(a^{0}, \chi_{1}^{0}\right) \times \\
& \int d^{4} x_{0} \sqrt{g\left(x_{0}\right)} C\left(\mu_{1}\right) f_{1}\left(\mu_{1}\right) C\left(\mu_{2}\right) f_{1}\left(\mu_{2}\right) .
\end{aligned}
$$

The action contains a surface term suitable for fixing an appropriate variable in the asymptotic region [10]. We can choose that variable so that it can be interpreted as an energy. Different choices of such an energy will lead to the selection of different vacua for the system, and will require the introduction of different surface terms in the action. Owing to these terms, it will not be possible in general to separate the action into two parts, one for the scale factor and another for the scalar field. However, there is a particular vacuum for which this separation is possible: the vacuum in which the associated solution for the scalar field is the conformal transform of the only solution damped at infinity when the background is flat; in this sense, this conformal vacuum is the conformal transform of the natural vacuum in flat space. Therefore, if $|0\rangle$ in Eq. (3.20) represents the conformal vacuum, the dependence on $a^{0}$ and $\chi_{1}^{0}$ in the integral separates. The part that depends on the conformal scalar field $\chi_{1}^{0}$ has the form

$$
\int d \chi_{1}^{0}\left(\chi_{1}^{0}\right)^{2} \mathcal{H}_{N_{1}} e^{-\left(\chi_{1}^{0}\right)^{2}}
$$

Since the $\mathcal{H}_{N_{1}}$ 's are the Hermite polynomials, this integral only gives a non-vanishing contribution when $N_{1}=0$ or $N_{1}=2$. The matrix element for $N_{1}=0$ represents a vacuum-to-vacuum transition without any interaction with the wormhole. Thus, in the conformal vacuum, the wormhole two-point function for the homogeneous mode $n=1$ finally becomes

$$
\left\langle 0\left|\phi\left(x_{1}\right) \phi\left(x_{2}\right)\right| \Psi_{N_{1}}\right\rangle=\delta_{2, N_{1}} \int d^{4} x_{0} \sqrt{g\left(x_{0}\right)} C\left(\mu_{1}\right) f_{1}\left(\mu_{1}\right) C\left(\mu_{2}\right) f_{1}\left(\mu_{2}\right) .
$$

We can interpret $\Psi_{N_{1}=2}$ as a wormhole state with two homogeneous scalar particles associated with the conformal vacuum. A similar analysis applied to three-point and higher functions would lead us to interpret the basis elements of the Hilbert space, $\Psi_{N_{1}}$, as wormhole states containing $N_{1}$ particles. For any other vacuum choice, there will exist a different orthonormal basis of the Hilbert space of wormholes such that only one basis element, apart from the vacuum itself, will give a non-vanishing contribution to the matrix element (3.22). In this situation, we could interpret this new basis element as a wormhole state with two particles associated with that specific vacuum. Therefore, the interpretation of wormholes as containing particles has the same ambiguity as the particle concept in quantum field theory in curved spacetimes. 
Taking into account Eq. (3.19), the matrix element for the mode $n=2$ is

$$
\begin{aligned}
& \left\langle 0\left|\phi\left(x_{1}\right) \phi\left(x_{2}\right)\right| \Psi_{N_{2}}\right\rangle=\int_{0}^{\infty} d a^{0} \int_{0}^{\infty} d \chi_{2}^{0}\left(\chi_{2}^{0}\right)^{2} \Delta\left(a^{0}, \chi_{2}^{0}\right) e^{-I^{s p}\left(a^{0}, \chi_{2}^{0}\right)} \Psi_{N_{2}}\left(a^{0}, \chi_{2}^{0}\right) \times \\
& \int d^{4} x_{0} \sqrt{g\left(x_{0}\right)} \int_{S O(4)} d h C\left(\mu_{1}\right) f_{2}\left(\mu_{1}\right) \mathcal{A}_{a_{1}}^{2} \widehat{x}_{1, x_{0} h}^{a_{1}} \mathcal{A}_{b_{1}}^{2} \widehat{x}_{2, x_{0} h}^{b_{1}} C\left(\mu_{2}\right) f_{2}\left(\mu_{2}\right) .
\end{aligned}
$$

The $S O(4)$ dependence of the integrand comes from the factor

$$
\mathcal{A}_{a_{1}}^{2} \widehat{x}_{1, x_{0} h}^{a_{1}} \mathcal{A}_{b_{1}}^{2} \widehat{x}_{2, x_{0} h}^{b_{1}}
$$

because $f_{2}\left(\mu_{1}\right)$ and $f_{2}\left(\mu_{2}\right)$ do not depend on the orientation. This can be re-expressed as

$$
\mathcal{A}_{a_{1}}^{2} R(h)_{c_{1}}^{a_{1}} \widehat{x}_{1, x_{0}}^{c_{1}} \mathcal{A}_{b_{1}}^{2} R(h)_{d_{1}}^{b_{1}} \widehat{x}_{2, x_{0}}^{d_{1}}
$$

where $R(h)_{c_{1}}^{a_{1}}$ denotes a rotation matrix in four dimensions, i.e. an irreducible unitary representation of the $S O(4)$ group. Using the property (see, e.g., Ref. 23])

$$
\int_{S O(4)} d h R(h)_{c}^{a} R(h)_{d}^{b}=\delta^{a b} \delta_{c d}
$$

we can straightforwardly carry out the average over orientations, obtaining

$$
\left(\mathcal{A}^{2} \cdot \mathcal{A}^{2}\right)\left(\widehat{x}_{1, x_{0}} \cdot \widehat{x}_{2, x_{0}}\right) .
$$

The product $\mathcal{A}_{a_{1}}^{2} \mathcal{A}^{2 a_{1}}$ is proportional to $\left(\chi_{2}^{0}\right)^{2}$, as can be seen from Eq. (3.16). Then, the matrix element (3.23) gives a non-vanishing result only in the case $N_{2}=0$ (vacuum-tovacuum transition) or $N_{2}=1$, that is, when there is a two-particle state associated with the conformal vacuum inside the wormhole. As in the case of homogeneous particles, the interpretation of different states of a wormhole as containing pairs of particles is related to the vacuum choice. We can write the final result for the matrix element between the conformal vacuum and a wormhole state as

$$
\left\langle 0\left|\phi\left(x_{1}\right) \phi\left(x_{2}\right)\right| \Psi_{N_{2}}\right\rangle=\delta_{1, N_{2}} \int d^{4} x_{0} \sqrt{g\left(x_{0}\right)} C\left(\mu_{1}\right) f_{2}\left(\mu_{1}\right)\left(\widehat{x}_{1, x_{0}} \cdot \widehat{x}_{2, x_{0}}\right) C\left(\mu_{2}\right) f_{2}\left(\mu_{2}\right) .
$$

For an arbitrary mode $n$, the factor that depends on orientations in Eq. (3.19) has the form

$$
\mathcal{A}_{a_{1} \cdots a_{n-1}}^{n} \widehat{x}_{1, x_{0} h}^{a_{1}} \cdots \widehat{x}_{1, x_{0} h}^{a_{n-1}} \mathcal{A}_{b_{1} \cdots b_{n-1}}^{n} \widehat{x}_{2, x_{0} h}^{b_{1}} \cdots \widehat{x}_{2, x_{0} h}^{b_{n-1}}
$$

In order to integrate this expression over $S O(4)$, we proceed as follows. First, we extract the $h$-dependence from each $\widehat{x}_{x_{0} h}^{b}$, i.e.

$$
\widehat{x}_{x_{0} h}^{a}=R(h)^{a}{ }_{b} \widehat{x}_{x_{0}}^{b} .
$$

Then, we absorb this $h$-dependence in the tensor $\mathcal{A}^{n}$ by forming a transformed tensor $\mathcal{A}_{h}^{n}$, which has the same symmetries as $\mathcal{A}^{n}$, because the linear space of tensors with such symmetries carries an irreducible tensor representation of the group $S O(4)$ and, therefore, this linear space is stable under rotations.

Second, using the symmetriser $S_{\mathcal{A}^{n}}$ corresponding to the symmetries of $\mathcal{A}^{n}$, we reexpress the product $\mathcal{A}_{h, a_{1} \cdots a_{n-1}}^{n} \widehat{x}_{x_{0}}^{a_{1}} \cdots \widehat{x}_{x_{0}}^{a_{n-1}}$ in the form

$$
\mathcal{A}_{h, a_{1} \cdots a_{n-1}}^{n} S_{\mathcal{A}^{n}}^{a_{1} \cdots a_{n-1}}
$$


with $S_{\mathcal{A}^{n}}^{a_{1} \cdots a_{n-1}}=S_{\mathcal{A}^{n}}\left(\widehat{x}_{x_{0}}^{a_{1}} \cdots \widehat{x}_{x_{0}}^{a_{n-1}}\right)$. As an illustration, for the mode $n=3, S_{\mathcal{A}^{n}}^{a_{1} a_{2}}$ is given by

$$
S_{\mathcal{A}^{n}}^{a_{1} a_{2}}=\left(\widehat{x}_{x_{0}}^{a_{1}} \widehat{x}_{x_{0}}^{a_{2}}-\frac{1}{4} \delta^{a_{1} a_{2}}\right) .
$$

Expression (3.31) is a scalar product in the linear space of tensors with the symmetries of $\mathcal{A}^{n}$. We will write it in the form $\mathcal{A}_{h}^{n} \cdot S_{\mathcal{A}^{n}}$. Since $\mathcal{A}_{h}^{n}=R(h) \mathcal{A}^{n}$, with $R(h)$ being in the appropriate irreducible representation of $S O(4)$, and

$$
\int_{S O(4)} d h R(h) \otimes R(h)=1 \otimes 1
$$

where $\otimes$ denotes the tensor product, the average over orientations of Eq. (3.29) results in

$$
\left(\mathcal{A}^{n} \cdot \mathcal{A}^{n}\right)\left(S_{1, \mathcal{A}^{n}} \cdot S_{2, \mathcal{A}^{n}}\right) .
$$

The term $\mathcal{A}^{n} \cdot \mathcal{A}^{n}$ is again proportional to $\left(\chi_{n}^{0}\right)^{2}$. Then, the matrix element for any mode $n>1$ in the conformal vacuum is

$$
\left\langle 0\left|\phi\left(x_{1}\right) \phi\left(x_{2}\right)\right| \Psi_{N_{n}}\right\rangle=\delta_{1, N_{n}} \int d^{4} x_{0} \sqrt{g\left(x_{0}\right)} C\left(\mu_{1}\right) f_{n}\left(\mu_{1}\right)\left(S_{1, \mathcal{A}^{n}} \cdot S_{2, \mathcal{A}^{n}}\right) C\left(\mu_{2}\right) f_{n}\left(\mu_{2}\right) .
$$

Finally, the matrix element (3.19) can be written as the sum of the calculated contributions of each mode. Indeed, the additional terms that involve cross products of different modes do not give any contribution because, in these cases, the average over orientations contains the vanishing integral

$$
\int_{S O(4)} d h R(h) \otimes 1=0
$$

\subsection{Electromagnetic field}

The matrix element for the electromagnetic wormhole interaction is given by the gauge invariant quantity

$$
\left\langle 0\left|A_{\mu}\left(x_{1}\right) A_{\nu}\left(x_{2}\right)\right| \Psi_{\alpha}\right\rangle .
$$

We choose the gauge in which the only degrees of freedom are the transverse waves and deal with each mode $A_{i}^{n}=\sum_{\sigma_{n}} \beta_{n \sigma_{n}} S_{i}^{n \sigma_{n}}$ separately. The Fadeev-Popov determinant associated with this gauge fixing condition is independent of the fields and can be reabsorbed in the normalisation [25]. The saddle point for the electromagnetic potential in adS spacetime must satisfy the equation

$$
(\square+3 \lambda) A_{\mu}^{n}=0
$$

In order to find solutions to this equation, we will follow the same steps as in the case of the scalar field. We first choose a cartesian basis in the degenerate space corresponding to each mode [17:

$$
A_{i}^{n}=\sum_{\sigma_{n}} \beta_{n \sigma_{n}}(\mu) \frac{\partial \widehat{x}^{a_{1}}}{\partial x^{i}} \mathcal{B}_{a_{1} a_{2} ; a_{3} \cdots a_{n}}^{n \sigma_{n}} \widehat{x}^{a_{2}} \widehat{x}^{a_{3}} \cdots \widehat{x}^{a_{n}} .
$$


Here, the $x_{i}$ 's are coordinates in the three-sphere and the functions $\beta_{n \sigma_{n}}$ satisfy the equation

$$
\ddot{\beta}_{n \sigma_{n}}+A \dot{\beta}_{n \sigma_{n}}-n^{2} C^{2} \beta_{n \sigma_{n}}=0,
$$

with $A(\mu)$ being given in Eq. (3.13); $\mathcal{B}_{a_{1} a_{2} ; a_{3} \cdots a_{n}}^{n \sigma_{n}}$ are the elements of an orthonormal basis in the space of tensors antisymmetric in their first two indices, symmetric with respect to all other indices, and that vanish when contracting any pair of indices or when taking a cyclic sum over $a_{1}, a_{2}$ and any other index. As we did with the scalar field, we will isolate all the $\mu$-dependence in just one variable for each mode,

$$
\beta_{n \sigma_{n}}(\mu)=\frac{\beta_{n \sigma_{n}}^{0}}{f_{n}\left(\mu^{0}\right)} f_{n}(\mu)
$$

where $\mu^{0}$ was defined in Eq. (3.14). We then define a new tensor,

$$
\mathcal{B}_{a_{1} a_{2} ; a_{3} \cdots a_{n}}^{n}=\sum_{\sigma_{n}} \frac{\beta_{n \sigma_{n}}^{0}}{f_{n}\left(\mu^{0}\right)} \mathcal{B}_{a_{1} a_{2} ; a_{3} \cdots a_{n}}^{n \sigma_{n}}
$$

which contains all the dependence on the arguments of the electromagnetic wormhole wave function.

The vector $A_{i}^{n}$ on the three-sphere can be expressed as a vector on adS spacetime by contracting it with $\frac{\partial x_{i}}{\partial x^{\mu}}$ :

$$
A_{\mu}^{n}(x)=f_{n}(\mu) \frac{1}{|\vec{x}|} \mathcal{B}_{a_{1} a_{2} ; a_{3} \cdots a_{n}}^{n}\left(\delta_{\mu}^{a_{1}}-\widehat{x}^{a_{1}} \widehat{x}^{b} \delta_{\mu b}\right) \widehat{x}^{a_{2}} \widehat{x}^{a_{3}} \cdots \widehat{x}^{a_{n}} .
$$

Owing to the antisymmetry of the tensor $\mathcal{B}^{n}$, we can sustitute the term inside the bracket by

$$
e_{\mu}^{a_{1}} \equiv \delta_{\mu}^{a_{1}}-\frac{\left[\left(1+\lambda \vec{x}^{2}\right)^{1 / 2}-1\right]}{\left(1+\lambda \vec{x}^{2}\right)} \widehat{x}^{a_{1}} \widehat{x}^{a} \delta_{a \mu} .
$$

In this way, the tensor character of $\mathcal{B}_{a_{1} a_{2} ; a_{3} \cdots a_{n}}^{n}$ under local changes of coordinates shows up explicitly. The tetrad $e_{\mu}^{a}$ defined here satifies the following relations, which we will use in what follows:

$$
\begin{gathered}
\frac{\partial x_{h}^{\nu}}{\partial x^{\mu}} e_{\nu}^{a}\left(\vec{x}_{h}\right)=R(h)^{a}{ }_{b} e_{\mu}^{b}, \\
\frac{\partial x_{x_{0}}^{\nu}}{\partial x^{\mu}} e_{\nu}^{a}\left(\vec{x}_{x_{0}}\right)=e_{\mu}^{a}, \\
g_{\mu \nu}=e_{\mu}^{a} e_{\nu}^{b} \delta_{a b} .
\end{gathered}
$$

The saddle point can still be separated into positive and negative helicity parts. This can be achived by introducing

$$
\mathcal{B}^{n \pm}=\frac{1}{2}\left(\mathcal{B}^{n} \pm{ }^{*} \mathcal{B}^{n}\right)
$$

where ${ }^{*} \mathcal{B}^{n}$ is the dual of the tensor $\mathcal{B}^{n}$ formed by contracting it with the Levi-Civita tensor

$$
{ }^{*} \mathcal{B}_{a_{1} a_{2} ; a_{3} \cdots a_{n}}^{n}=\epsilon_{a_{1} a_{2}}{ }^{c d} \mathcal{B}_{c d ; a_{3} \cdots a_{n}}^{n}
$$


The transformed saddle point for the mode $n$ and positive or negative helicity can be written, after some manipulations involving properties (3.45) and (3.46) of the tetrad, as

$$
\left(A_{x_{0} h}^{n \pm}\right)_{\mu}(x)=f_{n}(\mu) \frac{1}{\left|\vec{x}_{x_{0}}\right|} \mathcal{B}_{h, a_{1} a_{2} ; a_{3} \cdots a_{n}}^{n \pm} e_{\mu}^{a_{1}} \widehat{x}_{x_{0}}^{a_{2}} \widehat{x}_{x_{0}}^{a_{3}} \cdots \widehat{x}_{x_{0}}^{a_{n}}
$$

where we have absorbed all the $h$ dependence in the transformed tensor $\mathcal{B}_{h}^{n \pm}$. Once we notice that the $\mathcal{B}^{n \pm}$ 's carry an irreducible tensor representation of $S O(4)$ (as the $\mathcal{A}^{n}$ 's did in the scalar case), we can apply the same steps discussed in the previous subsection to carry out the average over orientation. The result of this average is proportional to

$$
\left(\mathcal{B}^{n \pm} \cdot \mathcal{B}^{n \pm}\right)\left(S_{1, \mathcal{B}^{n \pm} \mu} \cdot S_{2, \mathcal{B}^{n \pm} \nu}\right)
$$

with $S_{\mathcal{B}^{n \pm}}^{a_{1} a_{2} ; a_{3} \cdots a_{n}}=S_{\mathcal{B}^{n \pm}}\left(e_{\mu}^{a_{1}} \widehat{x}_{x_{0}}^{a_{2}} \widehat{x}_{x_{0}}^{a_{3}} \cdots \widehat{x}_{x_{0}}^{a_{n}}\right)$ being the symmetriser associated with the symmetries of $\mathcal{B}^{n \pm}$.

As in the inhomogeneous scalar field case, the matrix element between the conformal vacuum and a wormhole state is non-zero only when $N_{n}^{ \pm}=1$, i.e. when the wormhole contains a two-photon state in the mode $n$ with positive or negative helicity associated with the conformal vacuum, since the term $\mathcal{B}^{n \pm} \cdot \mathcal{B}^{n \pm}$ is proportional to $\left(\beta_{n}^{ \pm 0}\right)^{2}$.

At last, we can write the electromagnetic wormhole matrix element as

$$
\left\langle 0\left|A_{\mu}\left(x_{1}\right) A_{\nu}\left(x_{2}\right)\right| \Psi_{N_{n}^{ \pm}}\right\rangle=\delta_{1, N_{n}^{ \pm}} \int d^{4} x_{0} \sqrt{g\left(x_{0}\right)} C\left(\mu_{1}\right) f_{n}\left(\mu_{1}\right)\left(S_{1, \mathcal{B}^{n \pm} \mu} \cdot S_{2, \mathcal{B}^{n \pm} \nu}\right) C\left(\mu_{2}\right) f_{n}\left(\mu_{2}\right) .
$$

For the lowest mode $n=2$, the term inside the bracket is the Euclidean contraction of two tensors $S_{\mathcal{B}^{2 \pm} \mu}^{a_{1} a_{2}}$ of the form

$$
S_{\mathcal{B}^{2 \pm} \mu}^{a_{1} a_{2}}=\frac{1}{2}\left[\left(e_{\mu}^{a_{1}} \widehat{x}_{x_{0}}^{a_{2}}-e_{\mu}^{a_{2}} \widehat{x}_{x_{0}}^{a_{1}}\right) \pm \epsilon_{c d}^{a_{1} a_{2}}\left(e_{\mu}^{c} \widehat{x}_{x_{0}}^{d}-e_{\mu}^{d} \widehat{x}_{x_{0}}^{c}\right)\right]
$$

one evaluated at the point $x_{1}$ and the other at the point $x_{2}$.

These results have been obtained for the conformal vacuum, to which the wormhole basis (2.12) is related, although it is clear that we could have performed an equivalent analysis for any other choice of vacuum.

\subsection{Gravitons}

The techniques explained in the previous subsections can also be applied to gravitons in adS spacetime. In this case, the two-point funtions are

$$
\left\langle 0\left|h_{\mu \nu}\left(x_{1}\right) h_{\rho \sigma}\left(x_{2}\right)\right| \Psi_{\alpha}\right\rangle .
$$

The only non-vanishing elements of the metric perturbations, upon a suitable gauge fixation, are $h_{i j}=\sum_{n, \sigma_{n}} d_{n \sigma_{n}} G_{i j}^{n \sigma_{n}}$. The equation for $h_{\mu \nu}$ reads

$$
(\square+2 \lambda) h_{\mu \nu}=0 .
$$

Using a cartesian basis in the degenerate space corresponding to the mode $n$ [17], we can write

$$
h_{i j}=f_{n}(\mu) \frac{\partial \widehat{x}^{a_{1}}}{\partial x^{i}} \frac{\partial \widehat{x}^{a_{3}}}{\partial x^{j}} \mathcal{C}_{a_{1} a_{2} ; a_{3} a_{4} ; a_{5} \cdots a_{n+1}} \widehat{x}^{a_{2}} \widehat{x}^{a_{4}} \widehat{x}^{a_{5}} \cdots \widehat{x}^{a_{n+1}}
$$


where we have already isolated the $\mu$ dependence in just one variable $f_{n}$ satisfying now the equation

$$
\ddot{f}_{n}-A \dot{f}_{n}-\left(6 A^{2}-4 A C+C^{2}\left(n^{2}-3\right)\right) f_{n}=0 .
$$

The $\mathcal{C}^{n}$ 's, which contain the dependence on the arguments of the wave function, are tensors with the following properties: they are antisymmetric with respect to each pair of indices $a_{1} a_{2}$ and $a_{3} a_{4}$; symmetric with respect to all other indices, as well as under the interchange of the pair $a_{1} a_{2}$ with the pair $a_{3} a_{4}$; finally, they vanish when contracting any two indices or under cyclic sums over triplets of indices that contain the pair $a_{1} a_{2}$ or $a_{3} a_{4}$.

Now, we can find the transformed saddle point for the mode $n$ and positive or negative helicity:

$$
\left(h_{x_{0} h}^{n \pm}\right)_{\mu \nu}(x)=f_{n}(\mu) \frac{1}{\left|\vec{x}_{x_{0}}\right|^{2}} \mathcal{C}_{h, a_{1} a_{2} ; a_{3} a_{4} ; a_{5} \cdots a_{n+1}}^{n \pm} e_{\nu}^{a_{1}} \widehat{x}_{x_{0}}^{a_{2}} e_{\mu}^{a_{3}} \widehat{x}_{x_{0}}^{a_{4}} \widehat{x}_{x_{0}}^{a_{5}} \cdots \widehat{x}_{x_{0}}^{a_{n+1}}
$$

where

$$
\begin{gathered}
\mathcal{C}^{n \pm}=\left(\mathcal{C}^{n} \pm{ }^{*} \mathcal{C}^{n}\right) \\
{ }^{*} \mathcal{C}_{a_{1} a_{2} ; a_{3} a_{4} ; a_{5} \cdots a_{n+1}}^{n}=\frac{1}{2}\left(\epsilon_{a_{1} a_{2}}{ }^{c d} \mathcal{C}_{c d ; a_{3} a_{4} ; a_{5} \cdots a_{n+1}}^{n}+\epsilon_{a_{3} a_{4}}{ }^{c d} \mathcal{C}_{c d ; a_{1} a_{2} ; a_{5} \cdots a_{n+1}}^{n}\right) .
\end{gathered}
$$

Similarly to what happened in the electromagnetic case, the $\mathcal{C}^{n \pm}$ 's carry an irreducible tensor representation of the rotation group and, therefore, the average over orientations gives the result

$$
\left(\mathcal{C}^{n \pm} \cdot \mathcal{C}^{n \pm}\right)\left(S_{1, \mathcal{C}^{n \pm} \mu \nu} \cdot S_{2, \mathcal{C}^{n \pm} \rho \sigma}\right)
$$

in which we have introduced the symmetriser

$$
S_{\mathcal{C}^{n \pm} \mu \nu}^{a_{1} a_{2} ; a_{3} a_{4} ; a_{5} \cdots a_{n+1}}=S_{\mathcal{C}^{n \pm}}\left(e_{\mu}^{a_{1}} \widehat{x}_{x_{0}}^{a_{2}} e_{\nu}^{a_{3}} \widehat{x}_{x_{0}}^{a_{4}} \widehat{x}_{x_{0}}^{a_{5}} \cdots \widehat{x}_{x_{0}}^{a_{n+1}}\right) .
$$

The matrix elements between a vacuum and the elements $\Psi_{\alpha}$ of a basis of graviton wormhole states are

$$
\begin{aligned}
& \left\langle 0\left|h_{\mu \nu}\left(x_{1}\right) h_{\rho \sigma}\left(x_{2}\right)\right| \Psi_{\alpha}\right\rangle= \\
& K\left(0, \Psi_{\alpha}\right) \int d^{4} x_{0} \sqrt{g\left(x_{0}\right)} C^{2}\left(\mu_{1}\right) f_{n}\left(\mu_{1}\right)\left(S_{1, \mathcal{C}^{n \pm} \mu \nu} \cdot S_{2, \mathcal{C}^{n \pm} \rho \sigma}\right) C^{2}\left(\mu_{2}\right) f_{n}\left(\mu_{2}\right),
\end{aligned}
$$

where $K\left(0, \Psi_{\alpha}\right)$ is a constant that depends on the vacuum $|0\rangle$ and the state $\Psi_{\alpha}$. The vacuum $|0\rangle$ is specified by its associated mode expansion [22]. It is worth noting that, in general, these modes do not fall off at infinity [26] and, thus, the back-reaction effects of gravitons on the background diverge. Since there exists graviton creation in this system, we cannot interpret the states of the Hilbert space as wormholes containing certain number of gravitons.

Before we end this section, let us write the symmetriser $S_{\mathcal{C}^{n}}$ for the lowest mode $n=3$ as an example:

$$
S_{\mathcal{C}^{n} \mu \nu}^{a_{1} a_{2} a_{3} a_{4}}=R_{\mu \nu}^{a_{1} a_{2} a_{3} a_{4}}+\left(\delta^{a_{1}\left[a_{4}\right.} R_{\mu \nu}^{\left.a_{3}\right] a_{2}}+\delta^{a_{2}\left[a_{3}\right.} R_{\mu \nu}^{\left.a_{4}\right] a_{1}}\right)+\frac{1}{3} R_{\mu \nu}\left(\delta^{a_{1}\left[a_{3}\right.} \delta^{\left.a_{4}\right] a_{2}}\right)
$$


with

$$
\begin{aligned}
R_{\mu \nu}^{a_{1} a_{2} a_{3} a_{4}}= & \widehat{x}_{x_{0}}^{a_{1}} \widehat{x}_{x_{0}}^{a_{3}}\left(e_{\mu}^{a_{2}} e_{\nu}^{a_{4}}+e_{\mu}^{a_{4}} e_{\nu}^{a_{2}}\right)-\widehat{x}_{x_{0}}^{a_{1}} \widehat{x}_{x_{0}}^{a_{4}}\left(e_{\mu}^{a_{3}} e_{\nu}^{a_{2}}+e_{\mu}^{a_{2}} e_{\nu}^{a_{3}}\right)- \\
& \widehat{x}_{x_{0}}^{a_{2}} \widehat{x}_{x_{0}}^{a_{3}}\left(e_{\mu}^{a_{1}} e_{\nu}^{a_{4}}+e_{\mu}^{a_{4}} e_{\nu}^{a_{1}}\right)+\widehat{x}_{x_{0}}^{a_{2}} \widehat{x}_{x_{0}}^{a_{4}}\left(e_{\mu}^{a_{1}} e_{\nu}^{a_{3}}+e_{\mu}^{a_{3}} e_{\nu}^{a_{1}}\right) \\
R_{\mu \nu}^{a_{2} a_{4}}= & e_{\mu}^{a_{2}} e_{\nu}^{a_{4}}+e_{\mu}^{a_{4}} e_{\nu}^{a_{2}}+2 g_{\mu \nu} \widehat{x}_{x_{0}}^{a_{2}} \widehat{x}_{x_{0}}^{a_{4}}- \\
& n_{\mu} e_{\nu}^{a_{2}} \widehat{x}_{x_{0}}^{a_{4}}-n_{\nu} e_{\mu}^{a_{2}} \widehat{x}_{x_{0}}^{a_{4}}-n_{\mu} e_{\nu}^{a_{4}} \widehat{x}_{x_{0}}^{a_{2}}-n_{\nu} e_{\mu}^{a_{4}} \widehat{x}_{x_{0}}^{a_{2}} \\
R_{\mu \nu}= & 4\left(g_{\mu \nu}-n_{\mu} n_{\nu}\right) .
\end{aligned}
$$

In this expression, $n_{\mu} \equiv e_{\mu a} \widehat{x}_{x_{0}}^{a}$ is the normal in $\vec{x}$ to the three-sphere centered in $\vec{x}_{0}$.

\section{$4 \quad$ Effective interactions}

It has been proposed [2, 3] that the effects of baby universes branching off and joining onto a large universe can be modelled by adding local effective interaction terms to a quantum field theory in a fixed background. In this section, we will search for interaction Lagrangians $\mathcal{L}_{I}^{\alpha}$ that, via the formula

$$
\left\langle 0\left|\Phi\left(x_{1}\right) \Phi\left(x_{2}\right) \int d^{4} x_{0} \sqrt{g\left(x_{0}\right)} \mathcal{L}_{I}^{\alpha}\left(\Phi\left(x_{0}\right)\right)\right| 0\right\rangle,
$$

provide results equivalent to those calculated in Sec. 3. In this formula, $|0\rangle$ represents a matter field vacuum in adS background.

We will use the following ansatz for the interaction Lagrangian:

$$
\mathcal{L}_{I}=\Theta \Phi \cdot \Theta \Phi
$$

where $\Theta$ is a linear operator constructed with covariant derivatives $\nabla_{\rho}$, the adS metric $g_{\rho \sigma}$ and the Levi-Civita tensor. The dot in this expression means contraction of indices with the metric $g_{\rho \sigma}$. If we contract each spacetime index in $\Theta \Phi$ with the tetrads $e_{\rho}^{a}$, we transform a tensor under general coordinate transformations into a tensor under local changes of coordinates and the dot becomes Euclidean contraction.

Then, the linearity of the operator $\Theta$ and Wick's theorem allow us to write Eq. (4.1) in the form

$$
\int d^{4} x_{0} \sqrt{g\left(x_{0}\right)} \Theta^{\prime} G\left(x_{1}, x_{0}\right) \cdot \Theta^{\prime} G\left(x_{2}, x_{0}\right),
$$

where $G\left(x, x_{0}\right)$ is the propagator of the matter field $\Phi$. This propagator and all its covariant derivatives are bitensors invariant under the isometries of adS spacetime [16]. Primed indices (as well as the prime in $\Theta^{\prime}$ ) will be associated with the tangent space at the point $x_{0}$ of the adS background, while unprimed indices will be associated with the tangent space at the point $x$. The parallel transporter $g_{\mu \rho^{\prime}} \equiv e_{\mu}^{a} e_{\rho^{\prime}}^{a^{\prime}} \delta_{a a^{\prime}}$ connects these two tangent spaces. In this section, we will compare expression (4.3) with the matrix elements obtained in the previous section for a wormhole interacting with a conformal scalar field, photons and gravitons. From this comparison, we will deduce the form of the linear operator $\Theta$ and, consequently, the effective interaction Lagrangian for each matter content. 


\subsection{Conformal scalar field}

The propagator for a conformal scalar field in adS spacetime is an invariant biscalar satisfying the equation

$$
(\square+2 \lambda) G\left(x, x_{0}\right)=0 .
$$

It depends only on the geodesic distance $\mu\left(x, x_{0}\right)$ between the two points $x$ and $x_{0}$, an invariant quantity under the whole group of isometries of the adS geometry [16]. The explicit form of this propagator is determined by the choice of vacuum.

Notice that $G(\mu)$ is proportional to the homogeneous saddle point of the field $\phi$, since both of them depend only on $\mu$ and satisfy the same equation, namely, $\ddot{G}+3 A \dot{G}+2 \lambda G=0$. We can then write the two-point function for the homogeneous modem, Eq. (3.22), as

$$
\int d^{4} x_{0} \sqrt{g\left(x_{0}\right)} G\left(\mu_{1}\right) G\left(\mu_{2}\right) .
$$

Comparison with expression (4.3) shows that, in this case, $\Theta$ is the identity operator. Thus, the effective interaction Lagrangian that reproduces the effects of wormholes with two particles in the homogeneous mode is $\mathcal{L}_{I}(\phi)=\phi^{2}$.

Let us now analyse the mode $n=2$. It is convenient to introduce the normal at the point $x_{0}$ to the three-sphere centered in $x, n_{\rho^{\prime}} \equiv-e_{\rho^{\prime} a^{\prime}} \widehat{x}_{x_{0}}^{a^{\prime}}$, analogous to the normal $n_{\mu} \equiv e_{\mu a} \widehat{x}_{x_{0}}^{a}$ defined in Sec. 3. Naturally, these two normals are the covariant derivatives of the geodesic distance with respect $x_{0}$ and $x$, that is, $n_{\rho^{\prime}}=\nabla_{\rho^{\prime}} \mu$ and $n_{\mu}=\nabla_{\mu} \mu$. In view of these comments, it is worth noting that $-\widehat{x}_{x_{0}}^{a^{\prime}}$ and $\widehat{x}_{x_{0}}^{a}$ are the normals $n^{a^{\prime}}$ and $n^{a}$ in local coordinates. The parallel transporter in local coordinates is just $\delta_{a a^{\prime}}$, so the primed or unprimed character of the local coordinates is irrelevant.

It is easy to see that

$$
e_{\rho^{\prime}}^{a^{\prime}} \nabla^{\rho^{\prime}} G(\mu)=-\dot{G}(\mu) \widehat{x}_{x_{0}}^{a^{\prime}}
$$

and, moreover, that $\dot{G}$ satisfies the same equation as $C(\mu) f_{2}(\mu)$. Then, a term of the form

$$
\int d^{4} x_{0} \sqrt{g\left(x_{0}\right)} \nabla^{\prime} G\left(x_{1}, x_{0}\right) \cdot \nabla^{\prime} G\left(x_{2}, x_{0}\right)
$$

reproduces the result obtained for the matrix element (3.28) with a single two-particle state in the mode $n=2$ inside the wormhole. Comparison with Eq. (4.3) shows that $\Theta \phi=\nabla \phi$ and, thus, the effective interaction Lagrangian is $\nabla^{\rho} \phi \nabla_{\rho} \phi$.

For an arbitrary mode $n$, we need to find an operator $\Theta_{n}$ that, acting on the propagator, reproduces each of the two factors of Eq. (3.35), i.e. $\Theta_{n}^{\prime} G=C(\mu) f_{n}(\mu) S_{\mathcal{A}^{n}}$. Such operator turns out to be the only symmetric traceless linear combination with constant coefficients of all possible terms with $n-1$ indices that we can form using the primed metric and $n-1$ or fewer primed covariant derivatives. Let us prove this statement. The requirement that $\Theta_{n}^{\prime}$ be symmetric and traceless ensures that $\Theta_{n}^{\prime} G$ is the product of $S_{\mathcal{A}^{n}}$ and some function that depends only on the geodesic distance, denoted by $C(\mu) F_{n}(\mu)$ for convenience. This is so because $\Theta_{n}^{\prime} G$ is an invariant bitensor and, thus, it must be constructed only with the normal $n^{a^{\prime}}=-\widehat{x}_{x_{0}}^{a^{\prime}}$ and the metric $\delta^{a^{\prime} b^{\prime}}$ [16] in the same way as $S_{\mathcal{A}^{n}}$ was. We can also explicitly check that $\Theta_{n}^{\prime} G$ can be expressed in the form $C(\mu) F_{n}(\mu) S_{\mathcal{A}^{n}}$ by employing the relation between the derivative of the normal, the normal itself and the metric, which in local coordinates reads

$$
\nabla_{a^{\prime}} n_{b^{\prime}}=A(\mu)\left(\delta_{a^{\prime} b^{\prime}}-n_{a^{\prime}} n_{b^{\prime}}\right) .
$$


We will now show by induction that $F_{n}(\mu)$ satisfies the same equation as $f_{n}(\mu)$,

$$
\ddot{f}_{n}+A \dot{f}_{n}-n^{2} C^{2} f_{n}=0
$$

for any mode $n$. We have already seen that this is true for $n=1$. Let us assume that $\Theta_{n}^{\prime} G=C(\mu) f_{n}(\mu) S_{\mathcal{A}^{n}}$. Then, $\Theta_{n+1}^{\prime} G$, given by the symmetric traceless covariant derivative of $\Theta_{n}^{\prime} G$, becomes after some manipulations $C^{1-n} \frac{d}{d \mu}\left(C^{n} f_{n}\right) S_{\mathcal{A}^{n+1}}$. Finally, it is not difficult to check that $F_{n+1}=C^{-n} \frac{d}{d \mu}\left(C^{n} f_{n}\right)$ satisfies the same equation as $f_{n+1}$.

The application of this general prescription to the mode $n=3$ provides the operator $\Theta_{3}^{a b}=\nabla^{a} \nabla^{b}+\frac{1}{2} \lambda \delta^{a b}$. The interaction term associated with a wormhole containing a pair of scalar particles in the mode $n=3$ is, therefore,

$$
\left(\nabla^{\rho} \nabla^{\sigma} \phi+\frac{1}{2} \lambda g^{\rho \sigma} \phi\right)\left(\nabla_{\rho} \nabla_{\sigma} \phi+\frac{1}{2} \lambda g_{\rho \sigma} \phi\right)
$$

\subsection{Electromagnetic field}

The equation for the electromagnetic propagator in an adS background reads

$$
\left[g_{\mu \nu}(\square+3 \lambda)+(\zeta-1) \nabla_{\mu} \nabla_{\nu}\right] G^{\nu \rho^{\prime}}=0
$$

where $\zeta$ is a constant that specifies the gauge choice. For an arbitrary mode $n$, the bitensor $\left(\Theta_{n}^{\prime} G\right)_{\mu}^{a_{1}^{\prime} a_{2}^{\prime} ; a_{3}^{\prime} \cdots a_{n}^{\prime}}$ is given by a linear combination of terms of the form

$$
g^{a_{n}^{\prime} a_{n-1}^{\prime}} \cdots g^{a_{l+1}^{\prime} a_{l}^{\prime}} \nabla^{a_{l-1}^{\prime}} \ldots \nabla^{a_{2}^{\prime}} G_{\mu}^{a_{1}^{\prime}}
$$

The primed index structure of $\Theta_{n}^{\prime} G$ must be the same as that of $S_{\mathcal{B}^{n} \mu}^{a_{1}^{\prime} a_{2}^{\prime} ; a_{3}^{\prime} \cdots a_{n}^{\prime}}$, i.e. it must be antisymmetric in its first two indices, symmetric with respect to all other indices and must vanish when contracting any pair of indices or when taking a cyclic sum over $a_{1}, a_{2}$ and any other index. The proof of this statement goes along the same lines as that for the scalar field. The symmetry of $\Theta_{n}^{\prime} G$ ensures that $\Theta_{n}^{\prime} G=C(\mu) F_{n}(\mu) S_{\mathcal{B}^{n}}$ for some function $F_{n}(\mu)$. This funtion $F_{n}$ satisfies the same equation as the electromagnetic saddle point function $f_{n}$, provided that $F_{n-1}$ does, because $\Theta_{n}^{\prime}$ is constructed from $\Theta_{n-1}^{\prime}$ by taking its symmetric traceless covariant derivative as in the the scalar field case. It only remains to check that this is true for the lowest mode $n=2$. In this case,

$$
\left(\Theta_{2}^{\prime} G\right)_{\mu}^{a^{\prime} b^{\prime}}=\nabla^{a^{\prime}} G_{\mu}^{b^{\prime}}-\nabla^{b^{\prime}} G_{\mu}^{a^{\prime}}
$$

and can be rewritten as

$$
\left(\Theta_{2}^{\prime} G\right)_{\mu}^{a^{\prime} b^{\prime}}=C(\mu) F_{2}(\mu)\left(e_{\mu}^{a^{\prime}} \widehat{x}_{x_{0}}^{b^{\prime}}-e_{\mu}^{b^{\prime}} \widehat{x}_{x_{0}}^{a^{\prime}}\right)
$$

for some function $F_{2}(\mu)$. We define a new object $\left(\Theta_{2}^{\prime} G\right)_{\mu \nu}^{a^{\prime} b^{\prime}}=\nabla_{\nu}\left(\Theta_{2}^{\prime} G\right)_{\mu}^{a^{\prime} b^{\prime}}-\nabla_{\mu}\left(\Theta_{2}^{\prime} G\right)_{\nu}^{a^{\prime} b^{\prime}}$, which is equal to $\left\langle 0\left|F_{\mu \nu}(x) F^{a^{\prime} b^{\prime}}\left(x_{0}\right)\right| 0\right\rangle$, since $G_{\mu \rho^{\prime}}=\left\langle 0\left|A_{\mu}(x) A^{\rho^{\prime}}\left(x_{0}\right)\right| 0\right\rangle$. Therefore, it is gauge invariant and satisfies the field equation $\nabla^{\nu}\left(\Theta_{2}^{\prime} G\right)_{\mu \nu}^{a^{\prime} b^{\prime}}=0$. This translates into a second order differential equation for $F_{2}$, which is precisely the equation satisfied by $f_{2}(\mu)$ [16]. 
The interaction Lagrangian for the mode $n=2$ and positive and negative helicity can be obtained from the self-dual and anti-self-dual parts, $\Theta_{2+}$ and $\Theta_{2-}$, of the operator $\Theta_{2}$ given in Eq.. (4.13). These Lagrangians are

$$
\left(\Theta_{2 \pm} A\right)^{2}=\left(F \pm{ }^{*} F\right)^{2}
$$

Since $F_{\rho \sigma}{ }^{*} F^{\rho \sigma}$ is a topological invariant, both interaction Lagrangians reduce to $F^{2}$. Similarly, for an arbitrary mode $n$, the effective interaction terms corresponding to both helicities, $\left(\Theta_{n \pm} A\right)^{2}$, coincide because $\Theta_{n} A \cdot{ }^{*} \Theta_{n} A$ can be seen to be a topological invariant.

\subsection{Gravitons}

In this case, $\Theta_{n}^{\prime} G$ is a bitensor constructed with metrics and covariant derivatives with the same index structure and symmetries as the $S_{\mathcal{C}^{n}}$ 's. Owing to this structure, it has the form $C(\mu)^{2} F_{n}(\mu) S_{\mathcal{C}^{n}}$ for some function $F_{n}(\mu)$. In order to prove that $F_{n}(\mu)$ satisfies Eq. (3.57), we first note that $\Theta_{n}^{\prime} G=\left\langle 0\left|h(x) \Theta_{n}^{\prime} h\left(x_{0}\right)\right| 0\right\rangle$. In the gauge chosen in Sec. 3, $\Theta_{n}^{\prime} G$ satisfies

$$
(\square+2 \lambda) \Theta_{n}^{\prime} G=0,
$$

because $(\square+2 \lambda) h_{\mu \nu}=0$. Noting that $S_{\mathcal{C}^{n} \mu \nu}^{a_{1}^{\prime} a_{2}^{\prime} ; a_{3}^{\prime} a_{4}^{\prime} ; a_{5}^{\prime} \cdots a_{n}^{\prime}}$ are just the transverse traceless tensor harmonics $G_{i j}^{n \sigma_{n}}$ expressed in a cartesian basis for the degenerate space of mode $n$ and in spacetime coordinates, Eq. (4.1) becomes an equation for $F_{n}$, which is obviously Eq. (3.57). The interaction Lagrangian is then given by $\left(\Theta_{n \pm} h\right)^{2}$ for positive and negative helicities.

For the lowest mode $n=3, \Theta_{3} h$ is the Weyl tensor of the perturbation $h_{\mu \nu}$ (the Weyl tensor of the adS background vanishes). The Lagrangians associated with both helicities reduce then to $C_{\rho \sigma \eta \delta} C^{\rho \sigma \eta \delta}$, because the cross term $C_{\rho \sigma \eta \delta}{ }^{*} C^{\rho \sigma \eta \delta}$ is equal to the Pontryagin invariant [7].

\section{$5 \quad$ Summary and conclusions}

In this work, we have studied the effects of wormholes in low-energy physics in an adS background. We have calculated the wormhole matrix element between a vacuum and an arbitrary wormhole state, $\left\langle 0\left|\Phi\left(x_{1}\right) \Phi\left(x_{2}\right)\right| \Psi_{\alpha}\right\rangle$, explicitly and have found an effective interaction Lagrangian that reproduces this matrix element within the context of quantum field theory in adS background. As a first step, it has been necessary to find the Hilbert space of wormhole states. The invariance under the isotropy group $S O(4)$ only permits the existence of wormholes containing rotationally invariant matter states. For the inhomogeneous modes, this means that single-particle states are not allowed. We have considered scalar and electromagnetic fields, which are conformally coupled to gravity, and gravitons, which are not, because of the presence of a cosmological constant. In this case, the WDW equation cannot be separated but the wormhole Hilbert space can still be formally constructed in the semiclassical approximation.

We have seen that, for each vacuum choice, there exists an orthonormal basis in the Hilbert space of wormholes such that this matrix element vanishes for all basis states 
except for one of them and the vacuum itself. A similar analysis applies to three-point and higher functions. So, wormhole basis states can be labelled by the number of particles that they contain. Thus, the ambiguity in the choice of vacuum which is present in quantum field theory in curved spacetimes also shows up in wormhole physics. In the graviton case, we cannot label the wormhole states by their particle content because there is creation of gravitons owing to the expansion of the asymptotic region. The states are global states background-gravitons.

For each inhomogeneous mode $n$, the wormhole-induced effective interaction Lagrangian in adS spacetime is of the form

$$
\mathcal{L}_{I}=\left(\Theta_{n} \Phi\right)^{2},
$$

where the linear operator $\Theta_{n}$ can be constructed recursively by symmetrising $\nabla \Theta_{n-1}$ and substracting all its traces. Furthermore, for photons and gravitons, we have to deal with both helicities, positive and negative, separately. This amounts to introducing operators $\Theta_{n \pm}$ which are the self-dual and anti-self-dual parts of $\Theta_{n}$. We obtain the same interaction Lagrangian for positive and negative helicities, as expected, because the cross product $\Theta_{n} \Phi \cdot{ }^{*} \Theta_{n} \Phi$ is a topological invariant, as can be checked by direct calculation. The operator $\Theta_{n_{0}}$ for the lowest mode is specific to each matter content: it is the identity for the scalar field, the antisymmetric derivative in the electromagnetic case and, for gravitons, it has the same derivative structure as the Weyl tensor. Their associated Lagrangians read $\mathcal{L}_{I}=\phi^{2}$ for the scalar field, $\mathcal{L}_{I}=F_{\mu \nu} F^{\mu \nu}$ for photons and $\mathcal{L}_{I}=C_{\mu \nu \rho \sigma} C^{\mu \nu \rho \sigma}$ for gravitons, which coincide with the results obtained for asymptotically flat wormholes.

The interaction Lagrangians for higher modes in adS spacetime and those in the flat case differ in terms that are proportional to powers of the cosmological constant. For instance, the effective interaction Lagrangians coming from the $n=3$ inhomogeneous

mode of a scalar field is of the form $\left(\nabla^{\mu} \nabla^{\nu} \phi-\frac{1}{6} \Lambda g^{\mu \nu} \phi\right)^{2}$; similarly, we can easily find that the $n=4$ inhomogeneous mode of the electromagnetic field provides an effective interaction Lagrangian of the form $\left(\nabla^{\mu} \nabla^{\nu} F^{\rho \sigma}-\frac{1}{3} \Lambda g^{\mu \nu} F^{\rho \sigma}\right)^{2}$. It is also worth noting that adS wormholes do not induce any direct modification to the cosmological term nor to Newton's constant.

\section{Acknowledgments}

We are very grateful to Guillermo A. Mena Marugán, Mariano Moles and Pedro F. González-Díaz for helpful discussions and suggestions. C.B was supported by a Spanish Ministry of Education and Culture (MEC) grant. C.B. is also grateful to James Hartle and the Institute for Theoretical Physics (UCSB), where part of this work was done, for warm hospitality. This research was supported in part by the National Science Foundation under Grant No. PHY94-07194. L.J.G. was supported by funds provided by DGICYT and MEC under Contract Adjunct to the Project No. PB94-0107.

\section{References}

[1] A. Strominger, in Particles, strings and supernovae, edited by A. Jevicki and C. I. Tan (World Scientific, Singapore, 1989); S. W. Hawking, Mod. Phys. Lett. A5, 145 
(1990); ibid. A5, 453 (1990); L. J. Garay, Agujeros de gusano en cosmología cuántica, Ph. D. Thesis (Universidad Autónoma de Madrid, 1992) and references therein.

[2] S. Coleman, Nucl. Phys. B307, 867 (1988).

[3] S. W. Hawking, Nucl. Phys. B335, 155 (1990).

[4] S. W. Hawking, Phys. Rev. D 37, 904 (1988).

[5] A. Lyons, Nucl. Phys. B324, 253 (1989).

[6] H. F. Dowker, Nucl. Phys. B331, 194 (1990).

[7] H. F. Dowker and R. Laflamme, Nucl. Phys. B366, 209 (1991).

[8] L. J. Garay and J. García-Bellido, Nucl. Phys. B400, 416 (1993).

[9] A. Lukas, Nucl. Phys. B442, 533 (1995).

[10] C. Barceló, L. J. Garay, P. F. González-Díaz and G. A. Mena Marugán, Phys. Rev. D 53, 3162 (1996).

[11] C. Barceló, L.J. Garay, gr-qc/9703028, Phys. Rev. D (1998) in press.

[12] L. Klebanov, L. Susskind, T. Banks, Nucl. Phys. B317, 665 (1989).

[13] S. J. Avis, C. J. Isham and D. Storey, Phys. Rev. D 18, 3565 (1978).

[14] C. J. C. Burges, Nucl. Phys. B247, 533 (1984).

[15] B. Allen, Phys. Rev. D 32, 3136 (1985).

[16] B. Allen and T. Jacobson, Commun. Math. Phys. 103, 669 (1986).

[17] E.M. Lifshitz, J. Phys. 10, 116 (1946); E.M. Lifshitz and I. Khalatnikov, Adv. Phys. 12, 185 (1963).

[18] V. Moncrief, Phys. Rev. D 18, 983 (1978).

[19] M. Abramowitz and I.A. Stegun, Handbook of Mathematical Functions, Natl. Bur. Stand. Amppl. Math. Ser. No. 55, revised 9th. ed., edited by M. Abramowitz and I.A. Stegun, (U.S. Gort. Print. Off. Washington, D.C., 1970).

[20] L.P. Grishchuk, Zh. Eksp. Teor. Fiz. 67, 825 (1974); Sov. Phys. JETP 40, 409 (1975).

[21] J.J. Halliwell, in Proceedings of the Seventh Jerusalem Winter School for Theoretical Physics: Quantum Cosmology and Baby Universe, edited by S. Coleman, J. B. Hartle, T. Piran and S. Weinberg, (World Scientific. Singapore, 1990).

[22] S. Wada, Nucl. Phys. B276, 729 (1986).

[23] A. Barut and R. Raczka, Theory of Group Representations and Applications (World Scientific, Singapore, 1986). 
[24] S. Weinberg, Gravitation and Cosmology (John Wiley and Sons, 1972).

[25] P. Ramond, Field Theory: a Modern Primer (Addison-Wesley, New York, 1990).

[26] M. Turing, J. Math. Phys. 31, 669 (1990). 